\title{
Matter ejection and kilonova emission from binary neutron star mergers
}

\author{
Albino Perego*† \\ Istituto Nazionale di Fisica Nucleare, Sezione di Milano Bicocca, Piazza della Scienza 3, 20126 \\ Milano, Italia \\ Istituto Nazionale di Fisica Nucleare, Sezione di Milano Bicocca, Gruppo Collegato di Parma, \\ Parco Area della Scienza 7/A, 43124 Parma, Italia \\ E-mail: albino.perego@mib.infn.it
}

The first detection of GWs from a binary neutron star merger (GW170817) marked the beginning of the multimessenger astronomy era. A few hours after the GW detection, the observation of an associated electromagnetic counterpart compatible with a kilonova/macronova emission remarkably confirmed our basic picture concerning the ejection of matter and the nucleosynthesis occurring in such a kind of events. At the same time, it gave a first demonstration of the power of a multimessenger analysis in exploiting binary compact mergers as laboratory of fundamental physics. In this contribution, we will present the status of kilonova/macronova modeling in terms of the different ejection mechanisms, and of the associated $r$-process nucleosynthesis. We will show the impact of the variety of ejecta (both in terms of microphysical properties and spatial distributions) on the light curves, with a particular application to the case of GW170817. This modelling provides complementary information to the GW signal and is crucial to set multimessenger constraints, for example for the equation of state of nuclear matter.

GRAvitational-waves Science\&technology Symposium - GRASS2018

1-2 March 2018

Palazzo Moroni, Padova (Italy)

\footnotetext{
*Speaker.

${ }^{\dagger}$ A.P. acknowledges support from the University of Parma under the grant "FIL incentivanti".
} 


\section{Introduction}

A neutron star binary (BNS) is expected to merge due to the emission of gravitational waves (GWs). Despite the fact that the whole inspiral can last for billions of years, the final phases, corresponding to the last few orbits, to the merger, and to its aftermath, happen on a timescale of a few seconds. In the merger aftermath, the large reservoir of angular momentum produces an accretion disk of dense and hot matter around a central compact object. The latter could be a massive NS, possibly temporarily stabilized against gravitational collapse by differential rotation, or a black hole $(\mathrm{BH})$ promptly formed after merger. Due to the high temperatures and densities in the merger remnant, luminosities in excess of $10^{53} \mathrm{erg} \mathrm{s}^{-1}$ are radiated away in neutrinos of all flavors. In particular, the emission and the absorption of electron (anti)neutrinos via chargedcurrent reactions influence matter composition since they convert neutrons into protons and viceversa. The intense GW emission occurring during those final seconds makes BNS mergers one of the primary targets for ground-based GW detectors, like Advanced Ligo and Advanced Virgo [1, 2]. Prior to merger, each NS deforms due to the tidal field of the companion. The degree of deformation depends on the NS masses and on the still unknown equation of state (EOS) of NS matter [3, $4,5]$. Since the tidal deformability has a direct imprint on the GW signal, a detailed analysis of the signal coming from a BNS merger can provide unique information about the properties of matter around and above nuclear saturation density [6,7]. However, GWs are not the only messanger of BNS mergers. Due to its neutron richness, matter expelled during the merger is expected to undergo $r$-process nucleosynthesis and to potentially synthetize all heavy elements up to Uranium and Thorium. The radioactive decay of these freshly synthetized elements powers an electromagnetic (EM) transient called kilonova (or macronova). The precise composition of the ejecta, in addition to its amount and velocity, influences the properties of the kilonova light curve. Numerical models are the necessary tools to predict not only the GW signal, but also the nature of the remnant and the properties of the ejecta.

On August 17th, 2017, the detection of a GW signal compatible with the merger of two NSs (GW170817, [8]), in association with a short gamma-ray burst (GRB170817a, [9]) and a kilonova (AT2017gfo, [10]), marked the beginning of the multimessenger astronomy era. The kilonova was observed from ten hours up to a few weeks after merger by telescopes located all around the Earth and in space. The EM signal had an early blue component, peaking at one day in the ultraviolet and visible frequences, and evolved in a red component, characterized by peaks in the infrared frequences four days after. These first GW-EM combined detections gave us the unprecedent opportunity to test our understanding of BNS mergers and, at the same time, to exploit these events to probe the properties of dense matter in regimes that would never be accessible on terrestral laboratories.

\section{Matter ejection}

Matter ejection from merging BNS is expected to occur through different channels. In addition to the specific mechanism, each channel is characterized by its timescale and ejecta properties.

Dynamic ejecta Dynamical ejection happens for a few ms after merger due to tidal interactions between the NSs and shocks developing from the central remnant. The average expansion velocity 

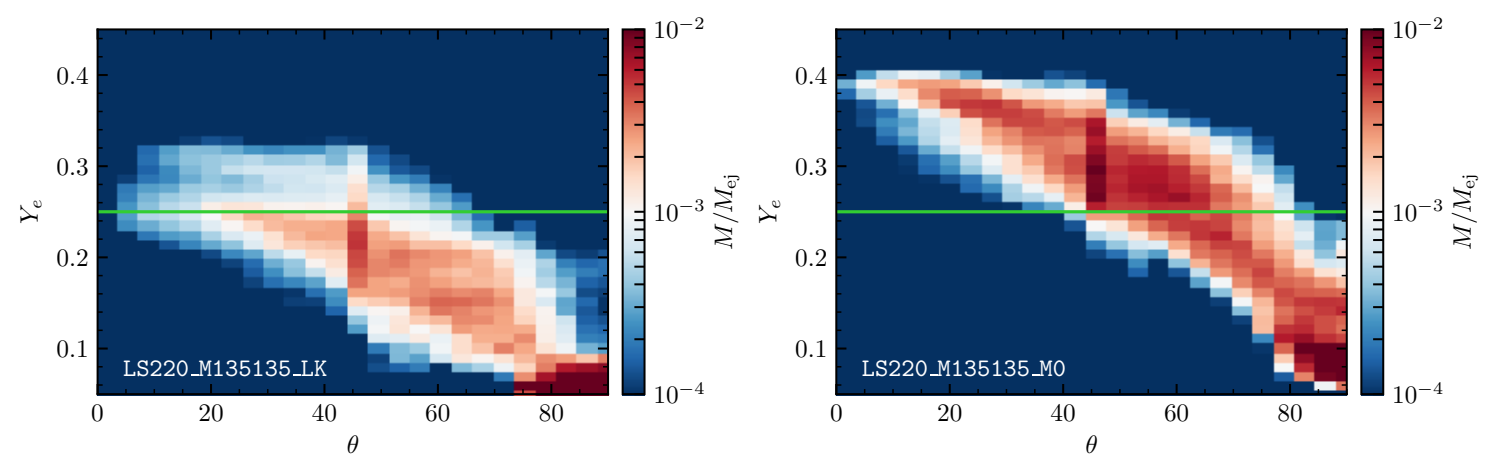

Figure 1: Color coded histograms of the distribution of the dynamical ejecta mass as a function of the polar angle and $Y_{\mathrm{e}}$ for two BNS merger simulations performed with the Lattimer-Swesty EOS with $K=220 \mathrm{MeV}$. In the simulation reported on the left side, only neutrino emission was taken into account in optically thin condition, while neutrino absoption was also included in the simulation on the right side [15].

is $\lesssim 0.3 c$, with a possible high velocity tail, and the amount of ejecta ranges between $10^{-4} M_{\odot}$ and $10^{-2} M_{\odot}$, depending on the properties of the binary and of the nuclear EOS $[11,12,13]$. Recent general relativistic hydrodynamical simulations including neutrinos revealed that the high temperatures reached during the merger and the intense neutrino irradiation can significantly change the composition of the ejecta $[14,15]$. In Fig. 1, we present histograms of the mass of dynamical ejecta as a function of the polar angle $(\theta)$ and of the final electron fraction $\left(Y_{\mathrm{e}}\right)$ for a $1.35 M_{\odot}-1.35 M_{\odot}$ BNS merger performed using the Lattimer-Swesty EOS. Matter is expelled at all latitudes, but with a peak along the equatorial plane and a minima along the polar axis. The inclusion of neutrino absorption processes in optically thin conditions drives the electron fraction above 0.25 for matter expanding close to the polar axis (right panel).
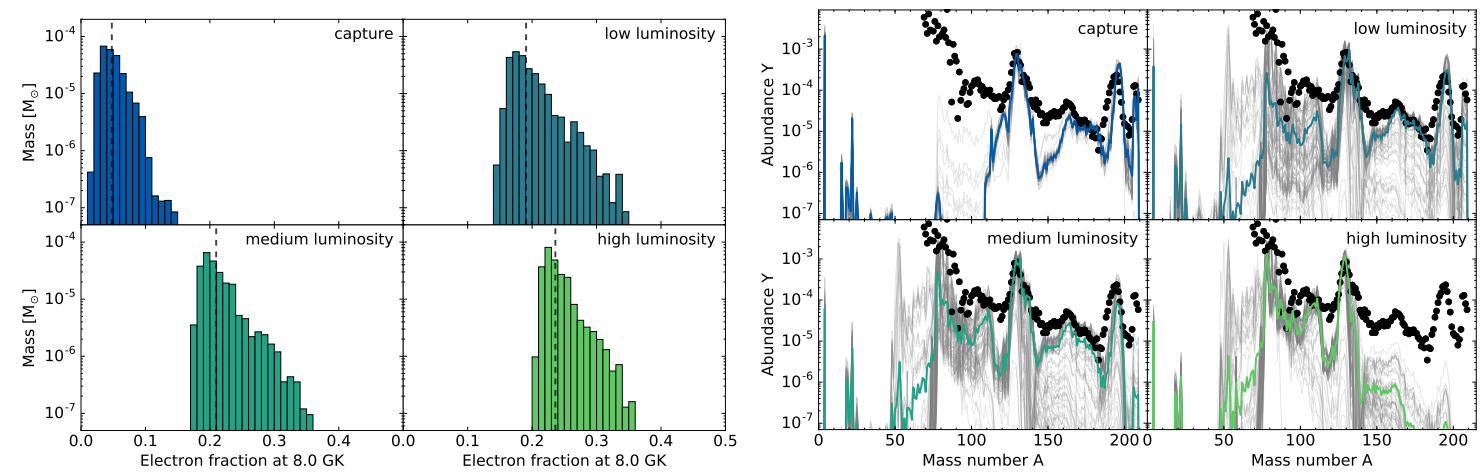

Figure 2: Distributions of the final electron fraction (left) and nucleosynthesis abundances (right) in the dynamical ejecta under the influence of weak processes. The four cases are detailed in [16]. Here we present the configuration where the initial $Y_{\mathrm{e}}$ is the one obtained by the original BNS merger simulation [17].

In [16] we analized in a post-processing study the evolution of $Y_{\mathrm{e}}$, entropy, and nuclear composition under the influence of neutrinos in the dynamical ejecta. The latter was obtained by a general relativistic merger simulation that did not include weak processes [17]. The parametrized neutrino luminosity allowed the study of the impact of neutrino absorption on the final ejecta composition. In Fig. 2, we report the distribution of the electron fraction (left panel) and of the nucleosynthesis 
abundances (right panel) obtained by post-processing the ejecta with a detailed nuclear network. Increasing neutrino luminosities drive the $Y_{\mathrm{e}}$ distribution toward larger average values. Electron (anti)neutrino luminosities in excess of $1.2(2.4) \times 10^{53} \mathrm{erg} \mathrm{s}^{-1}$ lead to $Y_{\mathrm{e}} \gtrsim 0.22$ for a large fraction of the ejecta, preventing the significant formation of nuclei behind the second $r$-process peak. These conditions are expected to occur more easily along the polar directions due to the anisotropic character of the neutrino emission, while very neutron rich matter is dynamically ejected along the equatorial plane. This possible bimodal distribution is crucial for the EM emission, since the production of Lanthanides and Actinides increases the photon opacity up to $\kappa \sim 10 \mathrm{~cm}^{2} \mathrm{~g}^{-1}[18,19]$, while in their absence the opacity is expected to stay closer to the one of Iron-group elements, $\kappa \sim 0.1 \mathrm{~cm}^{2} \mathrm{~g}^{-1}$.

Neutrino-driven winds After the formation of a disk around the central remnant, the energy deposition operated by neutrinos (mainly electron flavor ones) in the outer part of the disk drives the emission of matter from the disk in the form of a $v$-driven wind [20]. The timescale for this process to occur is of the order of a few tens of millisecond, comparable to the neutrino diffusion timescale from the innermost disk. This process was simulated in $3 \mathrm{D}$ using a Newtonian hydrodynamics code and a spectral leakage scheme [21]. The absorption of neutrinos drives $Y_{\mathrm{e}}$ above 0.3 , preventing the production of Lanthanides and Actinides in the part of the wind that becomes unbound once nuclear recombination has occurred [22]. This mechanism is more efficient in the presence of a long-lived massive NS, since the latter can contribute to the neutrino emission at least by the same amount as the disk [23]. On a timescale comparable to the disk lifetime, up to $5 \%$ of the disk can be expelled through this channel, preferentially toward the polar direction $(\theta \lesssim \pi / 3)$, with typical velocities $\lesssim 0.1 \mathrm{c}$. Winds of magnetic origin can develop on similar timescales as (or even in parallel to) $v$-driven winds [24]. If this matter is also subject to neutrino irradiation, the corresponding ejecta is expected to share similar properties and composition.

Viscous ejecta On the longer disk lifetime $(\sim 1 \mathrm{~s})$, viscosity of magnetic origin drives matter accretion on the central object but, at the same time, expands the outer part of the disk [25, 26]. As the temperature decreases due to neutrino cooling and matter expansion, neutrons and protons recombine to form nuclei. The released nuclear energy is able to unbind a significant fraction of the disk (up to a few tens of percent) with typical velocities $\lesssim 0.1 \mathrm{c}$ and moving away from the remnant in all directions . Due to the long ejection timescale, weak processes can re-set $Y_{\mathrm{e}}$ inside the disk, producing a wide distribution between $\sim 0.1$ and $\sim 0.45$, and consequently a varied composition of nuclei from the first to the third $r$-process peak $[27,28,29]$.

\section{Kilonova emission}

The large variety of properties that characterize the different ejection channels translates in a varied EM emission for the kilonova. To account for the ejecta properties and their angular distributions, we developed a multi-component, anisotropic kilonova model [30], based on the models presented in [31] and [22]. The polar angle is discretized in radial slices inside which radial models for the matter and photon expansion are computed. Three different ejection episodes are considered inside each ray, according to the ejecta properties summarized in the previous section. Thermal radiation powered by the radioactive decay of $r$-process elements is assumed to be emitted at the 

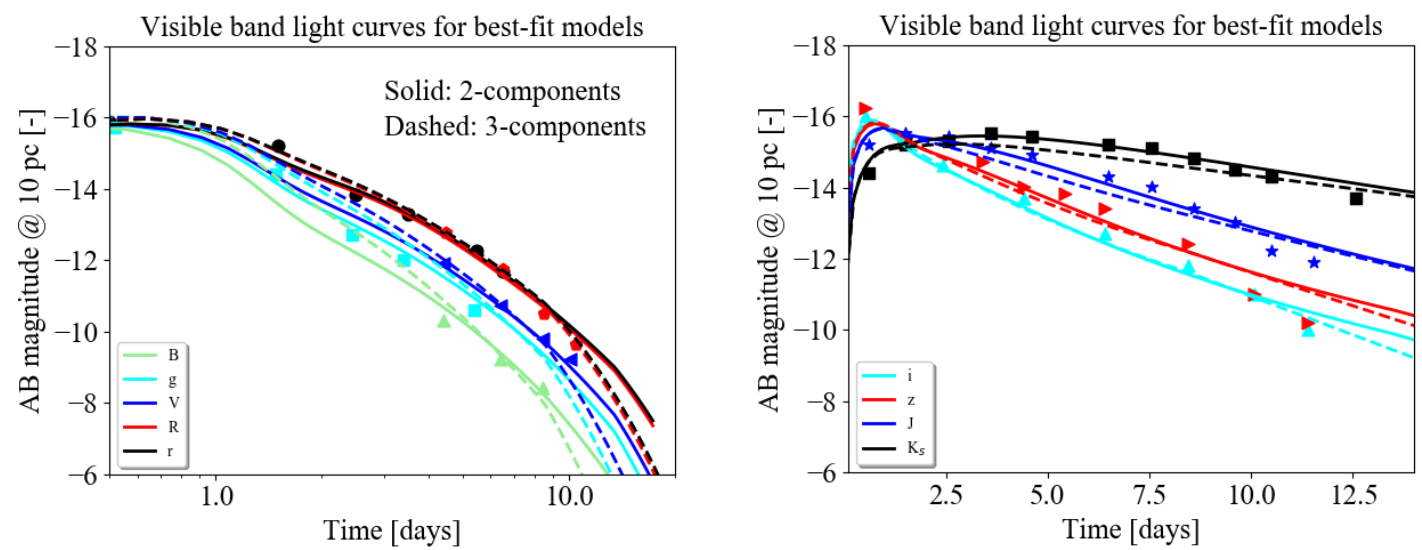

Figure 3: Selected light curves for visible (left) and infrared (right) filters for the best fit kilonova models obtained in [30] to reproduced observational data of AT2017gfo (markers, [32, 33]).

outermost photosphere. We computed the light curves in different photometric bands and compared with the AT2017gfo observations reported in [32] and [33] for the first 20 days after merger for ultraviolet, visible and infrared emissions. We produced light curves for a large sample of parameter sets, obtained by varying independently each of the model parameters inside fiducial ranges. Among the different parameter sets, we searched for the ones that showed the best agreement with observations. Light curves for the best-fit models are shown, alongside with observed data, in Fig. 3 for selected visible and infrared bands. According to our analysis, the observed kilonova emission is compatible with both two- and a three-component models. The polar axis of the merger was inclined with respect to the observer line-of-sight by $\sim 30^{\circ}$. The dynamical ejecta, amounting to $\sim 5 \times 10^{-3} M_{\odot}$, had a low opacity (blue) component at high latitude, moving with high speed $(0.25 c)$ in addition to a very opaque (red) equatorial component. Viscous and $v$-driven wind ejecta provide additional $\sim 0.45 M_{\odot}$ of ejected mass. The latter is characterized by low opacity $\left(\kappa \lesssim 1 \mathrm{~cm}^{2} \mathrm{~g}^{-1}\right)$, while the former by an intermediate one $\left(\kappa \sim 5 \mathrm{~cm}^{2} \mathrm{~g}^{-1}\right.$, purple).

\section{GW-EM joint constraints on the nuclear EOS}

The joint analysis of the GW and EM signals obtained from GW170817, in association with information extracted from general relativistic hydrodynamical simulations, could set genuine multimessenger constraints on the nuclear EOS [34]. On the one hand, the analysis of the GW signal provided an upper bound of 800 (at a 90\% confidence level) on the combined tidal parameter $\tilde{\Lambda}$ of the merging binary [8]. This dimensionless quantity describes the mutual deformation that each NS experiences during the latest stages of the inspiral due to the tidal field of the companion. Such a limit disfavors very stiff nuclear EOSs characterized by large deformabilities. On the other hand, the bright kilonova emission of AT2017gfo is compatible with a significant ejection of matter coming from the disk, as outlined in the previous section. A large sample of BNS merger simulations performed in numerical relativity, using different finite temperature EOSs, indicate that the merger of binaries characterized by $\tilde{\Lambda} \lesssim 400$ (because of either a soft EOS or two massive NSs) results in a prompt collapse of the remnant to a $\mathrm{BH}$. In this case, a disk massive enough to explain the kilonova emission cannot form. This is outlined in the left panel of Fig. 4, where the mass of the 

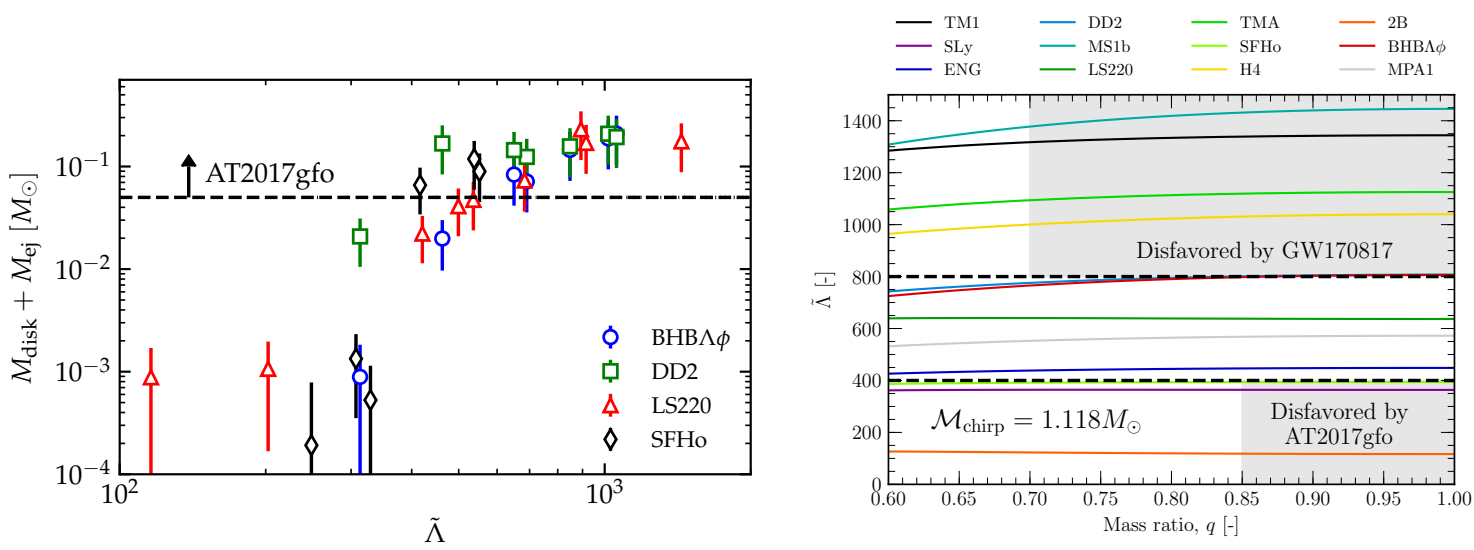

Figure 4: Left: sum of the disk and dynamical ejecta masses as a function of the $\tilde{\Lambda}$ parameter obtained for a large set of numerical relativity simulations of BNS mergers performed with four different finite temperature EOSs. Right: $\tilde{\Lambda}$ as a function of the binary mass ratio $q \equiv M_{\mathrm{A}} / M_{\mathrm{B}} \leq 1$ for a large set of EOSs, assuming a fixed chirp mass of $\mathscr{M}_{\text {chirp }}=1.188 \mathrm{M}_{\odot}$. The top (bottom) grey areas represent regimes excluded by GW170817 (AT2017gfo and GR simulations).

disk, incresed by the mass of the dynamical ejecta, is represented as a function of $\tilde{\Lambda}$ for simulations employing four different finite temperature EOSs. In the right panel of Fig. 4, we present theoretical curves of $\tilde{\Lambda}$ obtained for different EOSs and for BNS systems characterized by the chirp mass associated with $\mathrm{GW} 170817\left(1.188 \mathrm{M}_{\odot}\right)$, as a function of the binary mass ratio $q$. The lower bound deduced from the combined analysis of the EM signal and numerical relativity simulations seems to disfavor very soft EOSs and the prompt formation of a BH in GW170817.

\section{5. acknowledgement}

A.P. thanks S. Bernuzzi and D. Radice for useful discussion and help with the manuscript. A.P. acknowledges support from the INFN initiative "High Performance data Network" funded by CIPE.

\section{References}

[1] J. Aasi et al., Advanced LIGO, CQG 32 (2015) 074001

[2] F. Acernese et al., Advanced Virgo: a second-generation interferometric gravitational wave detector, CQG 32 (2015) 2

[3] T. Hinderer, Tidal Love Numbers of Neutron Stars, ApJ 677 (2008) 2

[4] T. Damour \& A. Nagar Effective One Body description of tidal effects in inspiralling compact binaries, PRD 81 (2010) 084016

[5] S. Bernuzzi et al., Tidal effects in binary neutron star coalescence, PRD 86 (2012) 044030

[6] J. Read et al., Measuring the neutron star equation of state with gravitational wave observations, $P R D 79$ (2009) 124033 
[7] D. Radice et al., Probing Extreme-Density Matter with Gravitational Wave Observations of Binary Neutron Star Merger Remnants, ApJ 842 (2016) L10

[8] B. P. Abbott et al., GW170817: Observation of Gravitational Waves from a Binary Neutron Star Inspiral, PRL 119 (2017) 161101

[9] B. P. Abbott et al., Gravitational Waves and Gamma-Rays from a Binary Neutron Star Merger: GW170817 and GRB 170817A, ApJ 848 (2017) L13

[10] B. P. Abbott et al., Multi-messenger Observations of a Binary Neutron Star Merger, ApJ 848 (2017) L12

[11] O. Korobkin et al., On the astrophysical robustness of neutron star merger r-process, MNRAS $\mathbf{4 2 6}$ (2012) 3

[12] K. Hotokezaka et al., The mass ejection from the merger of binary neutron stars, PRD 87 (2013) 024001

[13] A. Bauswein et al., Systematics of dynamical mass ejection, nucleosynthesis, and radioactively powered electromagnetic signals from neutron-star mergers, ApJ 773 (2013) 78

[14] Y. Sekiguchi et al., Dynamical mass ejection from binary neutron star mergers: Radiation-hydrodynamics study in general relativity, PRD 91 (2015) 064059

[15] D. Radice et al., Dynamical Mass Ejection from Binary Neutron Star Mergers, MNRAS 460 (2016) 3

[16] D. Martin et al., The role of weak reactions in dynamic ejecta from binary neutron star mergers, CQG 35 (2018) 3

[17] W. Kastaun et al, Structure of stable binary neutron star merger remnants: Role of initial spin, PRD 96 (2017) 043019

[18] D. Kasen et al., Opacities and spectra of the r-process ejecta from neutron star mergers, ApJ 774 (2013) 1

[19] M. Tanaka \& K. Hotokezaka, Radiative transfer simulations of neutron star merger ejecta, ApJ 775 (2013) 2

[20] A. Perego et al., Neutrino-driven winds from neutron star merger remnants, MNRAS 443 (2014) 4

[21] A. Perego et al., An Advanced Leakage Scheme for neutrino treatment in astrophysical simulations, ApJS 223 (2016) 22

[22] D. Martin et al., Neutrino-driven Winds in the Aftermath of a Neutron Star Merger: Nucleosynthesis and Electromagnetic Transients, ApJ $\mathbf{8 1 3}$ (2015) 2

[23] A. Perego et al., Neutrino annihilation above merger remnants: implications of a long-lived massive neutron star, JPhG $4 \mathbf{4}$ (2017) 084007

[24] D. Siegel et al., Magnetically Driven Winds from Differentially Rotating Neutron Stars and X-Ray Afterglows of Short Gamma-Ray Bursts, ApJ 785 (2014) L6

[25] B. D. Metzger et al., Time-dependent models of accretion discs formed from compact object mergers, MNRAS 390 (2008) 2

[26] R. Fernandez \& B. D. Metzger, Nuclear Dominated Accretion Flows in Two Dimensions. I. Torus Evolution with Parametric Microphysics, ApJ 763 (2013) 108

[27] O. Just et al., Comprehensive nucleosynthesis analysis for ejecta of compact binary mergers, MNRAS 448 (2015) 1 
[28] M.R. Wu et al., Production of the entire range of $r$-process nuclides by black hole accretion disc outflows from neutron star mergers, MNRAS 463 (2016) 3

[29] D. Siegel \& B. D. Metzger, Three-Dimensional General-Relativistic Magnetohydrodynamic Simulations of Remnant Accretion Disks from Neutron Star Mergers: Outflows and r-Process Nucleosynthesis, PRL 119 (2017) 23

[30] A. Perego et al., AT 2017gfo: An Anisotropic and Three-component Kilonova Counterpart of GW170817, ApJ 850 (2017) L37

[31] D. Grossman et al, The long-term evolution of neutron star merger remnants - II. Radioactively powered transients, MNRAS 439 (2014) 757

[32] E. Pian et al, Spectroscopic identification of r-process nucleosynthesis in a double neutron-star merger, Nature 551 (2017) 7678

[33] L. Tanvir et al, The Emergence of a Lanthanide-rich Kilonova Following the Merger of Two Neutron Stars, ApJ 848 (2017) L27

[34] D. Radice et al., GW170817: Joint Constraint on the Neutron Star Equation of State from Multimessenger Observations, ApJ 852 (2018) L2 